\title{
Hormonoterapia adyuvante en cáncer de mama. Revisión de la Evidencia y Abordaje Terapéutico en el Instituto Instituto Nacional de Cancerología, Bogotá, Colombia
}

\author{
Adjuvant hormonal therapy for breast cancer: Update of evidence and therapeutic \\ approach at the National Cancer Institute of Colombia
}

Ximena Briceño-Morales ${ }^{\mathrm{a}}$ (iD), Oswaldo Sánchez-Castillo ${ }^{\mathrm{b}}$ iD , Juan Carlos Velasquez ${ }^{\mathrm{b}}$, Carlos E. Bonilla González ${ }^{\mathrm{b}}$ (iD, Sergio Cervera-Bonillac ${ }^{\text {iD }}$, Luis Guzman-AbiSaab ${ }^{d}$, Ricardo Bruges-Maya ${ }^{b, f}$ iD , Javier Angel-Aristizabal ${ }^{c}$, Carlos Duarte $^{c}$ iD , Carlos Lehmann-Mosquera ${ }^{\mathrm{C}}$ (iD), Mauricio García-Mora ${ }^{\mathrm{e}}$ (iD, Fernando Contreras-Mejía ${ }^{\mathrm{b}}$ iD, Eduardo Rojas ${ }^{\mathrm{b}}$, Sandra E. Diaz-Casas ${ }^{\mathrm{C}}$ (D)

\begin{abstract}
Breast cancer is the most frequently diagnosed cancer in women around the planet. According to GLOBOCAN 2020, its incidence and mortality worldwide are calculated at 47.8 and 13.6 per 100,000 women, respectively; for Colombia, the estimated incidence rate is 48.3 per 100,000 women, while mortality is 13.1 per 100,000 women. About $70 \%$ of breast cancer expresses hormone receptors $(\mathrm{HR}+)$ and is amenable to management with hormonal therapy, which improves the clinical outcomes of patients in the adjuvant and metastatic settings. Specifically in the adjuvant setting, studies have shown that hormonal therapy reduces the risk of relapse by almost $50 \%$ and the risk of death by $30 \%$, after 10 years of follow-up. For this update, a literature search was carried out in two of the most important bibliographic databases, subsequently selecting the most relevant clinical trials, systematic reviews, and meta-analyses on the subject. Finally, joint meetings were held between the functional units for breast and soft tissue tumors and clinical oncology of the National Cancer Institute of Colombia (INC), which managed to establish the therapeutic approach for adjuvant treatment in preand postmenopausal patients with $\mathrm{HR}+$ breast cancer, considering each recommendation in relation to the risk of disease relapse.
\end{abstract}

Keywords: breast cancer, adjuvant therapy, hormonal antineoplastic agents, premenopause, postmenopause.

\section{Resumen}

El cáncer de mama es el cáncer más frecuentemente diagnosticado en las mujeres alrededor del planeta. Según GLOBOCAN 2020, su incidencia y mortalidad a nivel mundial están calculadas en 47.8 y 13.6 por cada 100 mil mujeres, respectivamente; para Colombia, la incidencia estimada es de 48.3 por cada 100 mil mujeres y la mortalidad de 13.1 por cada 100 mil mujeres. Cerca del $70 \%$ del cáncer de mama expresa receptores hormonales $(\mathrm{RH}+)$ y es suceptible de manejo con terapia hormonal, la cual mejora los desenlaces clínicos de las pacientes en los escenarios adyuvante y metastásico. Específicamente, en la adyuvancia los estudios han demostrado que la hormonoterapia disminuye en casi un $50 \%$ el riesgo de recaída y en un $30 \%$ el riesgo de muerte después de 10 años de seguimiento. Para esta actualización, se realizó una búsqueda de la literatura en dos de las bases de datos bibliográficas más influyentes, seleccionando posteriormente los ensayos clínicos, revisiones sistemáticas y metaanálisis más relevantes sobre el tema. Finalmente, se realizaron reuniones conjuntas entre las Unidades Funcionales de Seno y Tejidos Blandos y Oncología Clínica del Instituto Nacional de Cancerología de Colombia (INC), logrando establecer el abordaje terapéutico en el escenario adyuvante para las pacientes pre y posmenopáusicas con cáncer de mama $\mathrm{RH}+$, discriminando cada recomendación en relación al riesgo de recidiva.

Palabras clave: cáncer de mama, adyuvancia, agentes hormonales antineoplasicos, premenopausia, postmenopausia.

\footnotetext{
a. Especialista en entrenamiento en Mastología. FUCS - Instituto Nacional de Cancerología, Bogotá, Colombia

b. Especialista en Oncología Clínica. Unidad Funcional de Oncología Clínica. Instituto Nacional de Cancerología, Bogotá, Colombia

c. Especialista en Cirugía de Mama y Tumores de Tejidos Blandos. Unidad Funcional de Mama y Tumores de Tejidos Blandos. Instituto Nacional de Cancerología Bogotá, Colombia

d. Especialista en Mastología. Unidad Funcional de Mama y Tumores de Tejidos Blandos. Instituto Nacional de Cancerología, Bogotá, Colombia

e. Especialista en Cirugía Oncológica. Unidad Funcional de Mama y Tumores de Tejidos Blandos. Instituto Nacional de Cancerología, Bogotá, Colombia

f. Especialista en Oncología Clínica en Hospital San Ignacio, Centro Javeriano de Oncología, Bogotá, Colombia

Autor para correspondencia: Sandra E. Díaz Casas Correo electrónico: sdiaz@cancer.gov.co
} 


\section{Introducción}

La El cáncer de mama receptor hormonal positivo $(\mathrm{RH}+)$ representa el $70 \%$ del cáncer de mama diagnosticado en todo el mundo (1). La Guía de Práctica Clínica para la Detección Temprana, Tratamiento Integral, Seguimiento y Rehabilitación del Cáncer de Mama, publicada por el Ministerio de Salud y Protección Social de Colombia en el año 2013 (2), puntualizó las recomendaciones para el tratamiento hormonal adyuvante en pacientes pre y posmenopáusicas con cáncer de mama $\mathrm{RH}+$, sobre la base de la evidencia que había disponible hasta ese momento. Sin embargo, desde entonces han salido publicados numerosos ensayos clínicos que sugieren modificaciones en la conducta terapéutica para este grupo de pacientes, razón por la cual la Unidad Funcional de Mama y Tejidos Blandos del Instituto Nacional de Cancerología de Colombia (INC) tomó la decisión de revisar la literatura actualizada en el tema, hasta el año 2019, y hacer los ajustes pertinentes en el tratamiento hormonal adyuvante de las pacientes con cáncer de mama $\mathrm{RH}+$.

\section{Objetivos:}

1. Definir el tratamiento hormonal adyuvante para las pacientes con cáncer de mama no metastasico $\mathrm{RH}(+)$ en relación a su estado menopausico y a los factores de riesgo para recaída y muerte por la enfermedad en el Instituto Nacional de Cancerología.

2. Revisar la evidencia científica actualizada sobre hormonoterapia adyuvante en pacientes pre y posmenopáusicas con cáncer de mama $\mathrm{RH}+$.

\section{Justificación:}

- En pacientes con cáncer de mama receptor hormonal positivo $(\mathrm{RH}+)$, el riesgo de recurrencia de la enfermedad persiste hasta por 20 años (e incluso por el resto de la vida) después del diagnóstico inicial (3).

- El riesgo de recurrencia a distancia se correlaciona especialmente con el estado ganglionar y el tamaño tumoral (10-41\%) (3), por lo cual es indispensable establecer grupos de riesgo y definir el tipo de terapia hormonal adyuvante, así como su duración.

- Debido al mayor riesgo de recurrencia asociado con ganglios positivos y un tamaño tumoral mayor, en algunos subgrupos de pacientes se justifica el uso de terapia hormonal extendida con tamoxifeno o IA (4).

\section{Conceptos claves: (1)}

Cáncer de mama receptor hormonal positivo $(\mathrm{RH}+)$ : todo cáncer de mama en el que al menos el $1 \%$ $(>=1 \%)$ de las células tumorales expresan receptores hormonales (estrógeno, progesterona o ambos).

Hormonoterapia adyuvante: consiste en la administración de medicamentos antiestrogénicos después de la cirugía para el cáncer de mama, típicamente tamoxifeno en las pacientes premenopáusicas, e inhibidores de aromatasa o tamoxifeno en las posmenopáusicas.

Hormonoterapia adyuvante estándar: aquella terapia endocrina adyuvante que se administra por 5 años después de la cirugía, con opciones que varían según el estado menopáusico de las pacientes.

Hormonoterapia adyuvante extendida: consiste en la extensión de la adyuvancia endocrina más allá del año 5, por lo general hasta completar 7 a 10 años de hormonoterapia en total.

\section{Metodología}

Se realizó una búsqueda de los estudios primarios fase 3, revisiones sistemáticas y metaanálisis publicados en Pubmeb, Embase y Medline sobre hormonoterapia adyuvante en cáncer de mama receptor hormonal positivo, disponible hasta noviembre de 2020. Esta búsqueda incluyó una combinación de vocabulario controlado (Medical Subject Headings (MeSH), términos Emtree, DeCS, incluidos los términos explotados) y de términos de texto libre (considerando variantes de ortografía, sinónimos, acrónimos y truncamiento) para las siguientes palabras clave: "breast neoplasm", "adjuvant", "antineoplastic hormonal agents", "premenopause", "postmenopause"; con etiquetas de campo, operadores de proximidad y operadores booleanos. Dos de los autores (SEDC, XBM) revisaron independientemente todos los títulos y resúmenes para seleccionar únicamente los ensayos clínicos controlados, revisiones sistemáticas y metaanálisis más relevantes y extraer los datos. Luego se plasmó la revisión de la literatura en una presentación a cargo de la Especialista en Entrenamiento seleccionada por la UF de Mama y Tejidos Blandos (XBM), bajo 
la supervisión de uno de los docentes (SEDC) y se analizó la evidencia al interior del Servicio. Posteriormente, se llevó a cabo una reunión entre las UF de Mama y Tejidos Blandos y Oncología Clínica, en la cual se discutió nuevamente la evidencia con el fin actualizar las recomendaciones de manejo en torno a la hormonoterapia adyuvante en pacientes con cáncer de mama $\mathrm{RH}+$. Con relación a la indicación de supresión ovárica (SO) en las pacientes premenopáusicas, hubo discrepancias para definir la población que más se beneficia de esta estrategia, en comparación con los riesgos y potenciales eventos adversos asociados, por lo que en una segunda reunión se analizaron en detalle los estudios primarios existentes sobre este tema, en particular con sus apéndices suplementarios. Finalmente, se estableció el cambio en el abordaje terapéutico y se diseñaron dos flujogramas de manejo, que se discriminaron según el estado menopáusico de las pacientes (Figuras 1 y 2 ).

\section{Resumen de la evidencia:}

\section{- Pacientes premenopáusicas con cáncer de mama $\mathrm{RH}+$}

El metaanálisis del Early Breast Cancer Trialists' Collaborative Group (EBCTCG), publicado inicialmente en 1998 y actualizado en 2011 (5, 6), validó el manejo estándar con tamoxifeno adyuvante por 5 años en pacientes con cáncer de mama $\mathrm{RH}+$. El primer reporte incluyó todos los ensayos clínicos aleatorizados (ECA) iniciados antes de 1990, para un total de 55 estudios y cerca de 37 mil pacientes ( $\mathrm{N}$ 36.689) pre y posmenopáusicas con receptores de estrógenos positivos ( $\mathrm{RE}+$ ), negativos o desconocidos. En este metaanálisis se comparó la administración adyuvante de tamoxifeno por 1, 2 y 5 años vs. no tamoxifeno, así como diferentes dosis del medicamento $(20,30$ y $40 \mathrm{mg})$. Con un seguimiento a 10 años, aquellas pacientes con tumores con RE+o desconocidos, que recibieron tamoxifeno adyuvante, presentaron una reducción de la recidiva tumoral de $21 \%, 29 \%$ y $47 \%$, con 1,2 y 5 años de tamoxifeno, respectivamente $(2 p<0.00001)$. La reducción proporcional en el riesgo de morir fue de $12 \%, 17 \%$ y $26 \%$, respectivamente $(2 p=0.003)$. Para ambos desenlaces las diferencias fueron estadísticamente significativas, siendo mayor en pacientes con ganglios positivos e independiente del estado menopáusico. En cuanto a la recurrencia, el mayor beneficio del tamoxifeno adyuvante se encontró en los cinco primeros años desde el inicio del tratamiento, mientras que en términos de mortalidad el beneficio se produjo a lo largo de los diez primeros años. Como era de esperarse, la hormonoterapia con tamoxifeno se asoció con un incremento significativo en la incidencia de cáncer de endometrio $(2 p<0.00001)$, que se cuadruplicó con la adyuvancia por 5 años, pero el exceso de muertes por esta causa fue de tan sólo 1 a 2 por cada 1.000 mujeres en los primeros 10 años del seguimiento. Otras muertes por causas cardiacas o vasculares no fueron significativas en el grupo de pacientes que recibió tamoxifeno $(2 p>0.1)$. Finalmente, no se encontraron diferencias en cuanto a eficacia con dosis superiores a $20 \mathrm{mg} /$ día (5).

La actualización de este metaanálisis evaluó 20 estudios que incluyeron un total de 21.457 pacientes con tumores con RE positivo o negativo. La comparación fue tamoxifeno adyuvante por 5 años vs. no tamoxifeno. Con un seguimiento a 15 años, aquellas pacientes con tumores RE positivo que recibieron tamoxifeno adyuvante, presentaron una reducción en el riesgo de recidiva de $39 \%(2 p<0.00001)$ $y$ en el riesgo de muerte de $29 \%(p<0.0001)$. El estudio reafirmó la ausencia de beneficio del tamoxifeno en pacientes con tumores RE negativo. Nuevamente, la adyuvancia con tamoxifeno se asoció con incrementos muy pequeños pero significativos en la incidencia de cáncer de endometrio y tromboembolismo pulmonar, con un exceso de muertes por ambas causas no mayor al $1 \%$ durante los primeros 10 años de seguimiento (6).

Dos estudios avalaron la extensión de la terapia adyuvante con tamoxifeno en pacientes pre y posmenopáusicas con cáncer de mama y RE+. El primero de ellos, el estudio ATLAS (Adjuvant Tamoxifen: Longer Against Shorter), fue publicado en diciembre de 2012 y comparó la adyuvancia hormonal con tamoxifeno por 5 años vs. 10 años en 12.894 mujeres con cáncer de mama RE positivo, negativo o desconocido. En este estudio, cerca de la mitad de las participantes tenían tamaños tumorales mayores de $2 \mathrm{~cm}$ (53\%) y ganglios positivos (48\%). Con un seguimiento a 15 años, la terapia extendida con tamoxifeno demostró tener un impacto estadísticamente significativo en la disminución de la recurrencia del cáncer de mama (617 recurrencias vs. 711 recurrencias; $p=0.002$ ) y la mortalidad por esta causa (331 muertes vs. 397 muertes; $p=0.01$ ), con reducciones absolutas de $3.7 \%$ 
y $2.8 \%$, respectivamente. En pacientes con ganglios positivos, la estimación puntual de supervivencia libre de enfermedad (SLE) se encontró a favor del manejo con tamoxifeno por 10 años. La incidencia acumulada de cáncer de endometrio fue $1.6 \%$ y $3.1 \%$ con 5 y 10 años de tamoxifeno, respectivamente. El RR para el desarrollo de tromboembolismo pulmonar fue de 1.87 en las pacientes que recibieron tamoxifeno por 10 años. Tanto para cáncer endometrial como para tromboembolismo pulmonar, el incremento absoluto de la mortalidad fue $0.2 \%$. Resulta fundamental señalar que continuar el tamoxifeno por 10 años en lugar de suspenderlo a los 5 años reduce significativamente la recurrencia y la mortalidad únicamente después del año 10 desde el inicio del tamoxifeno, es decir, tras finalizar la hormonoterapia extendida (7).

El segundo estudio fue el aTTom (Adjuvant TamoxifenTo Offer More?) ( $\mathrm{N}$ 6.953), publicado poco después que el ATLAS y con un diseño metodológico muy similar. Sus resultados básicamente reafirmaron los ya mencionados, demostrando que la hormonoterapia adyuvante extendida con tamoxifeno reduce significativamente el riesgo de recurrencia del cáncer de mama (580 recurrencias vs. 672 recurrencias; $\mathrm{p}=0.003)$ y la mortalidad por esta misma causa ( 392 vs. 443; $p=0.05$ ). De nuevo, sólo después de los años 7 y 9 desde el inicio del consumo del tamoxifeno se pudieron observar reducciones significativas en términos de SLE y supervivencia global (SG) (8).

En 2018 se conocieron los resultados actualizados de los estudios SOFT (Suppression of Ovarian Function Trial) y TEXT (The Tamoxifen and Exemestane Trial) (9), con un seguimiento a 8 y 9 años, respectivamente. Estos son los dos ECA más importantes de manejo hormonal adyuvante con SO en pacientes premenopáusicas con cáncer de mama $\mathrm{RH}+$. El SOFT incluyó cerca de 3.000 participantes (N 3.047) con cáncer de mama operable, el 35\% de ellas tenían ganglios positivos y el $85 \%$ tumores HER2 negativo. En este estudio se aleatorizaron las participantes a recibir tamoxifeno, tamoxifeno más SO o exemestano más SO. Por su parte, TEXT incluyó algo más de 2.500 participantes (N 2.660) con cáncer de mama operable, el $48 \%$ de ellas tenían ganglios positivos y el $87 \%$ tumores HER2 negativo. En este segundo estudio se aleatorizaron las participantes a recibir tamoxifeno más SO o exemestano más SO. En el TEXT, la tasa estimada de SLE a 5 años fue $91.5 \%$ para el grupo de pacientes que recibió exemestano más SO vs. $87.3 \%$ para el grupo que recibió tamoxifeno más So, con diferencias estadísticamente significativas en cuanto a recurrencia y segundo cáncer invasivo (HR 0.72; IC 95\% 0.60-0.85), pero sin diferencias en términos de SG (HR 1.14; IC 95\% 0.86-1.51). En el SOFT, la tasa estimada de SLE a 8 años fue $78.9 \%$ para aquellas pacientes que recibieron tamoxifeno solo, $83.2 \%$ tamoxifeno más SO (HR 0.76; IC 95\% $0.62-0.93$ ) y $85.9 \%$ exemestano más SO (HR 0.65; IC $95 \%$ 0.53-0.81).

La tasa de supervivencia global a 8 años fue de $91.5 \%$ en el grupo del tamoxifeno solo, de $93.3 \%$ en el grupo de tamoxifeno más SO y de $92.1 \%$ en el grupo de exemestano más SO. Para este último desenlace, la diferencia fue estadísticamente significativa solo para la combinación tamoxifeno más SO vs. tamoxifeno solo (HR 0.67; IC 95\% 0.48-0.92). En el subgrupo de pacientes que recibió quimioterapia, la tasa de SLE a 8 años fue significativamente más alta para las pacientes que fueron llevadas a SO $(76.7 \%$ en el grupo de tamoxifeno más SO y $80.4 \%$ en el grupo de exemestano más SO). En este mismo subgrupo, la tasa de SG fue estadísticamente significativa solo para la combinación tamoxifeno más SO vs. tamoxifeno solo (HR 0.59; IC 95\% 0.42-0.84) (9).

El análisis combinado de los grupos que son comparables en los estudios SOFT y TEXT, mostró que la combinación exemestano más SO fue superior a la combinación tamoxifeno más SO en cuanto a SLE $(86.8 \%$ vs. $82.8 \% ; \mathrm{p}<0.001)$ y supervivencia libre de recurrencia a distancia (SLRD) $(91.8 \%$ vs. $89.7 \%$; $\mathrm{p}<0.02)$, pero no en términos de SG $(93.4 \%$ vs. $93.3 \%$; $\mathrm{p}<0.84$ ). El subgrupo de pacientes menores de 35 años demostró tener una tasa más alta de recurrencia a 5 años, con una mayor incidencia de enfermedad a distancia a 8 años. Para los dos desenlaces antes mencionados, el mayor beneficio en este grupo de pacientes ocurrió con la combinación exemestano más SO. Finalmente, los autores de este estudio recomiendan balancear los beneficios oncológicos de la SO con los posibles eventos adversos y la toxicidad asociada a una menopausia prematura: síntomas vasomotores, hipertensión, alteraciones musculoesqueléticas (incluyendo osteoporosis) e intolerancia a la glucosa, entre otros (9). En este escenario, el principal reto consiste en identificar adecuadamente los subgrupos de pacientes que pueden beneficiarse en mayor medida de la SO, con los más altos beneficios en recurrencia y mortalidad y menor tasa de eventos adversos. 


\section{- Pacientes posmenopáusicas con cáncer de mama $\mathrm{RH}+$}

En 2015, el EBCTCG publicó un metaanálisis que incluyó 31.920 pacientes posmenopáusicas con cáncer de mama temprano y $\mathrm{RH}+(10)$. El objetivo de este estudio fue comparar tres diferentes esquemas de tratamiento hormonal buscando diferencias en recurrencia y mortalidad por cáncer de mama. Para la comparación IA por 5 años vs. tamoxifeno por 5 años, los RR de recurrencia favorecieron significativamente los IA durante los años 0-1 (RR 0.64; IC 95\% 0.52-0.78) y 2-4 (RR 0.80; IC 95\% 0.680.93 ), siendo no significativos a partir de entonces. La mortalidad por cáncer de mama a 10 años fue menor con los IA que con tamoxifeno $(12.1 \%$ vs. $14.2 \%$; RR 0.85; IC 95\% 0.75-0.96). Para la comparación IA por 5 años vs. switch por 5 años (2-3 años de tamoxifeno más un IA hasta completar 5 años de tratamiento en total), la recurrencia se redujo significativamente sólo durante los años 0-1 (RR 0.74; IC 95\% 0.62-0.89), es decir, cuando los tratamientos difirieron, siendo similar durante los años 2-4 (RR 0.99; IC 95\% 0.851.15), cuando ambos grupos recibieron un IA. No hubo ningún efecto significativo adicional después del quinto año. La reducción de la mortalidad por cáncer de mama no fue significativa (RR 0.89; IC $95 \%$ 0.78-1.03). Por último, para la comparación switch por 5 años vs. tamoxifeno por 5 años, los RR de recurrencia favorecieron significativamente a los inhibidores de aromatasa durante los años 2-4 (RR 0.56; IC 95\% 0.46-0.67), pero no posteriormente. La mortalidad por cáncer de mama a 10 años fue menor con el cambio a IA que continuando la hormonoterapia únicamente con tamoxifeno hasta completar 5 años $(8.7 \%$ vs. $10.1 \% ; 2 p=0.015)(10)$.

Para todas las comparaciones, los RR de recurrencia favorecieron a los inhibidores de aromatasa durante el tiempo en que los tratamientos difirieron, pero no fueron significativos a partir de entonces, y la mortalidad por cáncer de mama se redujo mientras los tratamientos eran diferentes y posteriormente, y para todos los períodos combinados. Sin embargo, las características del tumor son importantes predictores del riesgo de recurrencia; por lo tanto, el efecto absoluto sobre los desenlaces al administrar un IA en lugar de tamoxifeno se observan en el análisis por subgrupos. Con relación al estado ganglionar, los riesgos de recurrencia a 5 años para las mujeres con enfermedad N0, N1 y N2 se redujeron significativamente en un $1.2 \%, 3.7 \%$ y $6.4 \%$, respectivamente $(10)$.
Con los resultados de este metaanálisis, queda claro que probablemente toda mujer posmenopáusica con cáncer de mama $\mathrm{RH}+$ se beneficia de un IA en algún momento de la adyuvancia hormonal, independientemente del estado ganglionar, aunque el efecto es más evidente en aquellas con ganglios positivos. Sin embargo, cualquier ganancia en SLE y SG debe sopesarse con los posibles eventos adversos de la terapia en la salud ósea y la función cognitiva, así como deberían tenerse también en cuenta las diferencias en costos. Algunas pacientes con un riesgo de recaída muy bajo podrían recibir solo tamoxifeno, puesto que el beneficio que obtendrían al incorporar un IA a su esquema de tratamiento quizá sea mínimo (11).

Existen numerosos ECA que han evaluado la extensión de la hormonoterapia con un IA más allá del año 5 en pacientes posmenopáusicas (12-23). Resulta difícil realizar comparaciones directas entre estos estudios ya que los diseños metodológicos, aunque similares, difieren en aspectos fundamentales como el tipo de hormonoterapia inicial (desde tamoxifeno, switch o un IA) y la duración total de la adyuvancia (7 o 10 años). Los estudios que compararon un IA vs. placebo por 2 a 5 años más, en pacientes que habían recibido tamoxifeno, terapia switch o un IA previamente, han mostrado distintos resultados (12, 15-19).

El estudio MA. $17(12,13)$ aleatorizó pacientes que habían recibido 5 años de tamoxifeno a continuar 5 años más con letrozol o placebo; se encontró una mejoría estadísticamente significativa en cuanto a SLE en el grupo de pacientes asignadas a letrozol (letrozol 93\% vs. placebo 87\%; $p<0.001$ ); sin embargo, no hubo diferencia en cuanto a SG entre los grupos. En un análisis por subgrupos no planificado de SLE, el efecto del letrozol fue al menos tan grande en las participantes con ganglios negativos (RR 0.47; $p=0,005$ ), como en aquellas con ganglios positivos (RR $0.60 ; p=0.003$ ). Este estudio fue actualizado en 2006, encontrando que en pacientes con ganglios positivos el letrozol extendido mejoró significativamente la SG, con una reducción en el riesgo de morir de $39 \%(p=0.04)$. Por su parte, el NSABP B-33 (National Surgical Adjuvant Breast and Bowel Project B-33) (16) no encontró diferencias en términos de SLE ni SG para los grupos exemestano vs. placebo por 5 años, en pacientes que habían recibido 5 años de hormonoterapia previa con tamoxifeno. No obstante, este estudio se cerró de forma temprana tras conocerse los resultados del 
primer análisis interino del MA 17. Como el 44\% de las participantes del grupo del placebo se pasaron al grupo del exemestano, resultó imposible predecir el efecto real del tratamiento extendido con el IA, ya que el análisis estadístico se realizó por intención a tratar, luego un gran porcentaje de las participantes que estaban recibiendo exemestano se analizaron en el grupo del placebo. Aun así, resulta interesante que el efecto del exemestano en SLE y supervivencia libre de recurrencia resultara estadísticamente significativo en el grupo de pacientes con tumores T2-3 y ganglios positivos.

Los estudios ABCSG 6a (Austrian Breast and Colorectal Cancer Study Group) y GIM4 (Gruppo Italiano Mammella) $(15,22)$ extendieron la hormonoterapia con IA hasta el año 7 (en promedio) en pacientes que habían recibido tamoxifeno previamente, encontrando beneficio en cuanto a SLE, no así en SG, para las pacientes que recibieron terapia hormonal extendida. Por el contrario, el estudio DATA (Extended Adjuvant Aromatase Inhibition After Sequential Endocrine Therapy) (18), que comparó 3 años vs. 6 años de anastrozol después de 2 o 3 años de manejo hormonal con tamoxifeno, encontró sólo mejoría en SLE en los subgrupos de pacientes con tamaños tumorales mayores de $2 \mathrm{~cm}$ y positividad para ambos receptores hormonales; pero la presencia de ganglios positivos no se asoció per se a un mayor beneficio con el anastrozol por 6 años. Estudios como el NSABP B-42 (19) y el AERAS (Arimidex Extended Adjuvant Randomized Study) (21) han reportado que recibir 10 años de adyuvancia hormonal, los cinco últimos con un IA (desde switch o un IA), es superior a recibir únicamente cinco años de hormonoterapia adyuvante (tamoxifeno, switch o IA). Sin embargo, otros estudios como el IDEAL (Optimal Duration of Extended Adjuvant Endocrine Therapy for Early Breast Cancer) (17) y el ABCSG 16 (20), que aleatorizaron pacientes que habían recibido cinco años de cualquier hormonoterapia previa, a recibir 2 o 5 años de terapia extendida con un IA, mostraron que la adyuvancia total por 10 años no es superior a la adyuvancia total por 7 años.

Los factores claves a considerar para la extensión de la terapia hormonal son un estadio más avanzado de la enfermedad, la edad temprana, las preferencias de las pacientes y la adherencia al tratamiento. Se debería considerar la terapia endocrina extendida para la mayoría de las mujeres pre y posmenopáusicas que recibieron quimioterapia, ya que es más probable que tengan un alto riesgo de recaída debido a los factores clínicos y patológicos convencionales (11).

Es importante resaltar que no existen estudios que hayan comparado específicamente la administración de 5 vs. 7 vs. 10 años de un IA únicamente, por lo que no es posible concluir de forma contundente que 7 o 10 años de un IA sean superiores a 5 años. Las mejores aproximaciones se tienen de los estudios en donde algún subgrupo de pacientes había recibido un IA como la única terapia hormonal previa antes de la aleatorización (17, 19-21). En este escenario, ocurre algo muy similar a lo antes mencionado, y es que 10 años de hormonoterapia son superiores a 5 años, pero no a 7 años. En cualquier caso, al ser tan heterogénea la terapia hormonal previa que se administró en estos estudios, no es clara la contribución específica que tiene el manejo exclusivo con IA más allá del año 5 , en los mejores resultados de estas pacientes.

\section{- Nuevos horizontes en pacientes pre y posmenopáusicas con riesgo intermedio y alto de recidiva tumoral}

Los inhibidores de kinasas dependientes de ciclinas (iCDK4/6) están aprobados para el manejo de las pacientes con cáncer de mama avanzado o metastásico $\mathrm{RH}+$, HER2-. El uso de estos medicamentos, en conjunto con la terapia hormonal, demostraron mejorar significativamente la supervivencia libre de progresión (SLP) y la SG en este contexto (24-31). Por lo anterior, los iCDK4/6 se han convertido en objeto de investigación en el escenario adyuvante. En 2020 se publicó el estudio monarchE (Abemaciclib Combined With Endocrine Therapy for the Adjuvant Treatment of HR+, HER2-, Node-Positive, High-Risk, Early Breast Cancer), un ensayo clínico controlado fase III que incluyó hombres y mujeres pre y posmenopáusicas con más de 4 ganglios positivos, o de 1 a 3 ganglios positivos y al menos una de las siguientes características: tamaño tumoral $>=5 \mathrm{~cm}$, grado histológico 3 o Ki $67>=20 \%$. Entre julio de 2017 y agosto de 2019, 5.637 pacientes fueron aleatorizados a recibir hormonoterapia más abemaciclib vs. hormonoterapia sola. El tratamiento con abemaciclib se administró por 2 años y la duración total de la hormonoterapia fue de 5 a 10 años. El $43 \%$ de las participantes eran premenopáusicas y el $95 \%$ habían recibido quimioterapia (37\% en el escenario neoadyuvante). Para la fecha del segundo análisis interino, la hormonoterapia más abemaciclib 
Tabla 1. Resumen de la evidencia: Terapia hormonal adyuvante en pacientes premenopáusicas

\begin{tabular}{|c|c|c|c|c|}
\hline Estudio & Participantes & Comparación & \multicolumn{2}{|c|}{ Desenlaces } \\
\hline \multicolumn{5}{|c|}{ Terapia estándar con tamoxifeno (Tam*) } \\
\hline $\begin{array}{l}\text { Metaanálisis } \\
\text { EBCTCG } \\
1998\end{array}$ & $\begin{array}{l}\text { - Ca. de mama } \\
\text { temprano } \\
\text { - 36.689 pacientes } \\
\text { (55 ECAs) } \\
\text { - RE }+/-0 \\
\text { desconocidos } \\
\text { - Pre y } \\
\text { posmenopáusicas }\end{array}$ & $\begin{array}{c}\text { Tam por 1, } 2 \text { o } 5 \text { años } \\
\text { Vos. Tam }\end{array}$ & $\begin{array}{c}\text { Recurrencia: } \\
\text { Tam por } 5 \text { años disminuye } \\
\text { el riesgo de recurrencia en } \\
\text { un } 47 \%\end{array}$ & $\begin{array}{c}\text { Mortalidad: } \\
\text { Tam por } 5 \text { años disminuye el } \\
\text { riesgo de morir en un } 26 \%\end{array}$ \\
\hline $\begin{array}{l}\text { Metaanálisis } \\
\text { EBCTCG } \\
2011\end{array}$ & $\begin{array}{l}\text { - Ca. de mama } \\
\text { temprano } \\
\text { - 21.457 pacientes } \\
\text { (20 ECAs) } \\
\text { - RE+/- }\end{array}$ & \multirow[t]{2}{*}{$\begin{array}{l}\text { Tam por } 5 \text { años } \\
\text { Vs. } \\
\text { No tamoxifeno }\end{array}$} & $\begin{array}{c}\text { Recurrencia: } \\
\text { Tam por } 5 \text { años disminuye } \\
\text { el riesgo de recurrencia en } \\
\text { un } 39 \%\end{array}$ & $\begin{array}{l}\text { Mortalidad: } \\
\text { Tam por } 5 \text { años disminuye el } \\
\text { riesgo de morir en un } 30 \%\end{array}$ \\
\hline & $\begin{array}{c}\text { - Pre y } \\
\text { posmenopáusicas }\end{array}$ & & \multicolumn{2}{|c|}{$\begin{array}{c}\text { En tumores RH- no hay beneficio con la administración de } \\
\text { Tam }\end{array}$} \\
\hline \multicolumn{5}{|c|}{ Terapia extendida con tamoxifeno (Tam*) } \\
\hline ATLAS & $\begin{array}{c}\text { - Ca. de mama } \\
\text { temprano } \\
-12.894 \text { pacientes } \\
\text { - RE }+/-0 \\
\text { desconocidos } \\
\text { - Pre y } \\
\text { posmenopáusicas }\end{array}$ & $\begin{array}{l}\text { Tam por } 5 \text { años } \\
\text { Vs. } \\
\text { Tam por } 10 \text { años }\end{array}$ & $\begin{array}{c}\text { Recurrencia: } \\
\text { La disminución absoluta } \\
\text { en el riesgo de recurrencia } \\
\text { con Tam por } 10 \text { años es de } \\
3.7 \%\end{array}$ & $\begin{array}{c}\text { Mortalidad: } \\
\text { La disminución absoluta en el } \\
\text { riesgo de morir con Tam por } 10 \\
\text { años es de } 2.8 \%\end{array}$ \\
\hline \multirow[t]{2}{*}{ aTTOm } & $\begin{array}{l}\text { - Ca. de mama } \\
\text { temprano } \\
-6.953 \text { pacientes } \\
\text { - } \mathrm{RE}+/-\end{array}$ & \multirow[t]{2}{*}{$\begin{array}{l}\text { Tam por } 5 \text { años } \\
\text { Vs. } \\
\text { Tam por } 10 \text { años }\end{array}$} & $\begin{array}{c}\text { Recurrencia: } \\
\text { La disminución absoluta } \\
\text { en el riesgo de recurrencia } \\
\text { con Tam por } 10 \text { años es de } \\
1.9 \%\end{array}$ & $\begin{array}{l}\text { Mortalidad: } \\
\text { La disminución absoluta en el } \\
\text { riesgo de morir con Tam por } 10 \\
\text { años es de } 4 \%\end{array}$ \\
\hline & $\begin{array}{c}\text { - Pre y } \\
\text { posmenopáusicas }\end{array}$ & & \multicolumn{2}{|c|}{$\begin{array}{l}\text { La disminucion en el riesgo de recurrencia y muerte varían de } \\
\text { acuerdo al periodo de tiempo evaluado (pj. 5-10 años, 10-15 } \\
\text { años, > }>15 \text { años) }\end{array}$} \\
\hline \multicolumn{5}{|c|}{ Supresión ovárica (SO**) } \\
\hline \multirow{2}{*}{ SOFT } & $\begin{array}{l}\text { - Ca. de mama } \\
\text { temprano } \\
\text { - } 3.047 \text { pacientes }\end{array}$ & $\begin{array}{l}\text { Tam } \\
\text { Vs. } \\
\text { Tam }+ \text { so } \\
\text { V/s }\end{array}$ & \multicolumn{2}{|c|}{ Análisis combinado } \\
\hline & $\begin{array}{l}\text { premenopausicas } \\
-\mathrm{RH}+>=10 \% \\
-35 \% \mathrm{G}+\end{array}$ & $\begin{array}{l}\text { Exemestano }+\mathrm{SO} \\
\text { - Tratamiento por } 5 \text { años }\end{array}$ & \multirow{2}{*}{$\begin{array}{c}\text { Recurrencia: } \\
\text { En comparación con } \\
\text { Tam solo, Tam + SO y } \\
\text { Exemestano + SO reducen } \\
\text { entre un } 3.5 \% \text { y un } 5.2 \% \\
\text { el riesgo absoluto de } \\
\text { presentar una recurrencia } \\
\text { a distancia } \\
\\
\text { Para las pacientes que no } \\
\text { reciben QT }{ }^{* * *} \text { el beneficio } \\
\text { en recurrencia a distancia } \\
\text { es sólo con la combinación } \\
\text { Exemestano + SO }\end{array}$} & \multirow[b]{2}{*}{$\begin{array}{c}\text { Mortalidad: } \\
\text { Sólo la combinación Tam + So } \\
\text { genera un beneficio significativo } \\
\text { en mortalidad (para la población } \\
\text { general, mejoria de } 1.8 \% \text { en la } \\
\text { tasa de supervivencia global } \\
\text { con Tam + SO Vs. Tam solo; y } \\
\text { 4.3\% para las pacientes que } \\
\text { recibieron QT }{ }^{* * *} \text { ) }\end{array}$} \\
\hline TEXT & $\begin{array}{l}\text { - Ca. de mama } \\
\text { temprano } \\
\text { - } 2.660 \text { pacientes } \\
\text { premenopáusicas } \\
\text { - } \mathrm{RH}+>=10 \% \\
\text { - } 48 \% \mathrm{G}+\end{array}$ & $\begin{array}{c}\text { Tam + aGnRH } \\
\text { Vs. } \\
\text { Exemestano }+ \text { aGnRH } \\
\text { - Tratamiento por } 5 \text { años }\end{array}$ & & \\
\hline
\end{tabular}

* Tam = tamoxifeno

** SO = supresión ovárica = aGnRH (triptorelina), ablación ovárica actínica o quirúrgica

*** QT=quimioterapia 
Tabla 2. Resumen de la evidencia: Terapia hormonal adyuvante en pacientes posmenopáusicas

\begin{tabular}{|c|c|c|c|}
\hline Estudio & Participantes & Comparación & Desenlaces \\
\hline \multirow{3}{*}{$\begin{array}{c}\text { Metaanálisis } \\
\text { EBCTCG } \\
2015\end{array}$} & \multirow{3}{*}{$\begin{array}{l}\text { - Ca. de mama temprano } \\
\text { - } 31.920 \text { pacientes pos } \\
\text { menopáusicas } \\
\text { - RE+ }\end{array}$} & $\begin{array}{l}\text { IA* por } 5 \text { años } \\
\text { Vs. } \\
\text { Tam por } 5 \text { años }\end{array}$ & $\begin{array}{c}\text { Recurrencia: } \\
\text { - Años 0-1: RR } 0.64 \text { (IC 95\% 0.52-0.78) } \\
\text { - Años 2-4: RR 0.80 (IC } 95 \% 0.68-0.93 \text { ) } \\
\text { - Sin diferencia a partir del año } 5 \\
\text { Mortalidad: } \\
\text { - 12.1\% Vs. } 14.2 \% \text {; RR } 0.85 \text { (IC } 95 \% 0.75-0.96 \text { ) }\end{array}$ \\
\hline & & $\begin{array}{c}\text { IA por } 5 \text { años } \\
\text { Vs. } \\
\text { Switch** }(5 \text { años de terapia } \\
\text { hormonal en total) }\end{array}$ & $\begin{array}{c}\text { Recurrencia: } \\
\text { - Años 0-1: RR 0.74 (IC 95\% 0.62-0.89) } \\
\text { - Años 2-4: RR 0.99 (IC } 95 \% 0.85-1.15) \\
\text { - Sin efecto significativo adicional partir del año } 5 \\
\text { Mortalidad: } \\
\text { - RR 0.89 (IC 95\% 0.78-1.03) }\end{array}$ \\
\hline & & $\begin{array}{l}\text { Switch ( } 5 \text { años de terapia } \\
\text { hormonal en total) } \\
\text { Vs. } \\
\text { Tam por } 5 \text { años }\end{array}$ & $\begin{array}{c}\text { Recurrencia: } \\
\text { - Años 2-4: RR 0.56 (IC } 95 \% \text { 0.46-0.67) } \\
\text { - Sin diferencia a partir del año } 5 \\
\text { Mortalidad: } \\
-8.7 \% \text { Vs. } 10.1 \%(2 \mathrm{p}=0.015)\end{array}$ \\
\hline
\end{tabular}

\section{Terapia extendida con IA}

\begin{tabular}{|c|c|c|c|c|}
\hline MA. 17 & $\begin{array}{c}\text { - } 5.187 \text { pacientes pos } \\
\text { menopáusicas } \\
-\mathrm{G}^{* * *}-/+(50 \%+) \\
-\mathrm{RH}+\text { o desconocidos } \\
\text { - } 5 \text { años de manejo previo con } \\
\text { Tam }\end{array}$ & $\begin{array}{l}\text { Letrozol por } 5 \text { años } \\
\text { Vs. } \\
\text { Placebo por } 5 \text { años }\end{array}$ & $\begin{array}{c}\text { Recurrencia: } \\
\text { SLE: HR } 0.57 \text { (IC 95\% 0.43- } \\
\text { 0.75); a favor de Letrozol } \\
\text { por } 5 \text { años }\end{array}$ & $\begin{array}{l}\text { Mortalidad: } \\
\quad \text { Sin diferencia } \\
\text { Actualización, 2006: } \\
\text { En paciente con G+, } \\
\text { Letrozol por } 5 \text { años: } \\
\text { 39\% de reducción en } \\
\text { el riesgo de morir } \\
\qquad(\mathrm{p}=0.04)\end{array}$ \\
\hline MA. 17R & $\begin{array}{c}\text { - } 1.918 \text { pacientes pos } \\
\text { menopáusicas } \\
\text { - Cualquier T y N (47.5\% G+) } \\
\text { - RH+ o desconocidos } \\
\text { - } 5 \text { años de manejo previo con } \\
\text { un IA }\end{array}$ & $\begin{array}{l}\text { Letrozol por } 5 \text { años más } \\
\text { Vs. } \\
\text { Placebo por } 5 \text { años más } \\
\text { - En total } 10 \text { años de } \\
\text { tratamiento con un IA }\end{array}$ & $\begin{array}{c}\text { Recurrencia: } \\
\text { SLE: HR } 0.66 \text { (IC } 95 \% \text { 0.48- } \\
0.91 \text { ); a favor de Letrozol } \\
\text { por } 5 \text { años más }\end{array}$ & $\begin{array}{l}\text { Mortalidad: } \\
\text { Sin diferencia }\end{array}$ \\
\hline MA. 17R & $\begin{array}{c}\text { - } 1.918 \text { pacientes pos } \\
\text { menopáusicas } \\
\text { - Cualquier T y N (47.5\% G+) } \\
\text { - RH+ o desconocidos } \\
\text { - } 5 \text { años de manejo previo con } \\
\text { un IA }\end{array}$ & $\begin{array}{l}\text { Letrozol por } 5 \text { años más } \\
\text { Vs. } \\
\text { Placebo por } 5 \text { años más } \\
\text { - En total } 10 \text { años de } \\
\text { tratamiento con un IA }\end{array}$ & $\begin{array}{c}\text { Recurrencia: } \\
\text { SLE: HR } 0.66 \text { (IC 95\% 0.48- } \\
\text { 0.91); a favor de Letrozol } \\
\text { por } 5 \text { años má }\end{array}$ & $\begin{array}{l}\text { Mortalidad: } \\
\text { Sin diferencia }\end{array}$ \\
\hline NSABP B-33 & $\begin{array}{c}\text { - } 1.598 \text { pacientes pos } \\
\text { menopáusicas } \\
-\mathrm{T} 1-3 \mathrm{~N} 0-1(48 \% \mathrm{G}+) \\
-\mathrm{RH}+ \\
-5 \text { años de manejo previo con } \\
\begin{array}{c}\text { Tam } \\
\text { mame }\end{array}\end{array}$ & $\begin{array}{c}\text { Exemestano por } 5 \text { años } \\
\text { Vs. } \\
\text { Placebo por } 5 \text { años }\end{array}$ & $\begin{array}{c}\text { Recurrencia: } \\
\text { SLE: RR 0.68 ( } p=0.07) ; \text { a } \\
\text { favor de Exemestano por } \\
5 \text { años } \\
\text { Mayor beneficio en SLE en } \\
\text { pacientes T2-3 y G+ }\end{array}$ & $\begin{array}{l}\text { Mortalidad: } \\
\text { Sin diferencia }\end{array}$ \\
\hline IDEAL & $\begin{array}{c}\text { - } 1.821 \text { pacientes pos } \\
\text { menopáusicas } \\
\text { - Ca. de mama temprano y } \mathrm{G}+/ \text { - } \\
(64 \% \mathrm{G}+) \\
-\mathrm{RH}+ \\
\text { - Manejo previo con cualquier } \\
\text { terapia hormonal por } 5 \text { años }\end{array}$ & $\begin{array}{l}\text { Letrozol por } 2.5 \text { años } \\
\text { Vs. } \\
\text { Letrozol por } 5 \text { años }\end{array}$ & $\begin{array}{c}\text { Recurrencia: } \\
\text { SLE: HR } 0.92 \text { (IC 95\% 0.74- } \\
\text { 1.16); sin diferencia }\end{array}$ & $\begin{array}{l}\text { Mortalidad: } \\
\text { Sin diferencia }\end{array}$ \\
\hline DATA & $\begin{array}{c}\text { - } 1.912 \text { pacientes pos } \\
\text { menopáusicas } \\
-\mathrm{T} 1-2(92 \%) \\
-\mathrm{G}+/-(68 \% \mathrm{G}+) \\
-\mathrm{RH}+ \\
-2-3 \text { años de manejo previo } \\
\text { con Tam }\end{array}$ & $\begin{array}{l}\text { Anastrozol por } 3 \text { años } \\
\text { Vs. } \\
\text { Anastrozol por } 6 \text { años }\end{array}$ & $\begin{array}{c}\text { Recurrencia: } \\
\text { SLE: HR } 0.79 \text { (IC } 95 \% \text { 0.62- } \\
\text { 1.02); sin diferencia }\end{array}$ & $\begin{array}{l}\text { Mortalidad: } \\
\text { Sin diferencia }\end{array}$ \\
\hline
\end{tabular}




\begin{tabular}{|c|c|c|c|c|}
\hline ABCSG 16 & $\begin{array}{c}-3.484 \text { pacientes pos } \\
\text { menopáusicas } \\
- \text { E I-III } \\
- \text { RH+ } \\
-5 \text { años de cualquier terapia } \\
\text { hormonal previa }\end{array}$ & $\begin{array}{l}\text { Anastrozol por } 2 \text { años } \\
\text { Vs. } \\
\text { Anastrozol por } 5 \text { años }\end{array}$ & $\begin{array}{c}\text { Recurrencia: } \\
\text { SLE: HR 1.007 (IC 95\% 0.87- } \\
\text { 1.16); sin diferencia }\end{array}$ & $\begin{array}{l}\text { Mortalidad: } \\
\text { Sin diferencia }\end{array}$ \\
\hline NSABP B-42 & $\begin{array}{c}\text { - } 3.966 \text { pacientes pos } \\
\text { menopáusicas } \\
\text { - E I-IIIA ( } 43 \% \mathrm{G}+) \\
-\mathrm{RH}+ \\
-5 \text { años de manejo previo con } \\
\text { IA o desde el switch con Tam } \\
(<=3 \text { años) }\end{array}$ & $\begin{array}{l}\text { Letrozol por } 5 \text { años } \\
\text { Vs. } \\
\text { Placebo por } 5 \text { años }\end{array}$ & $\begin{array}{c}\text { Recurrencia: } \\
\text { SLE: HR } 0.85 \text { (IC } 95 \% \text { 0.73- } \\
\text { 0.99); sin diferencia } \\
\text { Actualización, } 2019: \\
\text { SLE: HR=0.84 (IC } 95 \% \text { 0.74- } \\
0.96 \text { ); a favor de Letrozol } \\
\text { por } 5 \text { años }\end{array}$ & $\begin{array}{l}\text { Mortalidad: } \\
\text { Sin diferencia }\end{array}$ \\
\hline $\begin{array}{c}\text { AERAS } \\
\text { (abstract) }\end{array}$ & $\begin{array}{c}\text { - } 1.697 \text { mujeres pos } \\
\text { menopáusicas } \\
- \text { E I-III } \\
- \text { RH+ } \\
-5 \text { años de manejo previo con } \\
\text { anastrozol o desde el switch } \\
\text { con Tam }\end{array}$ & $\begin{array}{c}\text { Anastrozol por } 5 \text { años } \\
\text { Vs. } \\
\text { No tratamiento adicional }\end{array}$ & $\begin{array}{c}\text { Recurrencia: } \\
\text { SLE: } 91.9 \% \text { Vs. } 84.4 \% \text {; } \\
\text { p=0.004; a favor de } \\
\text { Anastrozol por } 5 \text { años } \\
\text { SLE a distancia: } 97.2 \% \text { Vs. } \\
\text { 94.3\%; } p=0.0077 ; \text { a favor } \\
\text { de Anastrozol por } 5 \text { años }\end{array}$ & $\begin{array}{l}\text { Mortalidad: } \\
\text { Sin diferencia }\end{array}$ \\
\hline $\begin{array}{c}\text { GIM4 } \\
\text { (abstract) }\end{array}$ & $\begin{array}{c}\text { - } 2.056 \text { mujeres pos } \\
\text { menopáusicas } \\
\text { - Ca. de mama temprano (56\% } \\
\text { G-) } \\
- \text { RH+ } \\
\text { - Manejo previo con Tam por } 2-3\end{array}$ & $\begin{array}{l}\text { Letrozol por 2-3 años } \\
\text { Letrozol por } 5 \text { años }\end{array}$ & $\begin{array}{c}\text { Recurrencia: } \\
\text { SLE: HR } 0.82 \text { (IC 95\% 0.68- } \\
\text { 0.98); a favor de Letrozol } \\
\text { por } 5 \text { años }\end{array}$ & $\begin{array}{l}\text { Mortalidad: } \\
\text { Sin diferencia }\end{array}$ \\
\hline
\end{tabular}

* IA = inhibidor de aromatasa

** Swicht = manejo inicial con 2 o 3 años de Tam y posteriormente 2 o 3 años de IA

${ }^{* * *} \mathrm{G}=$ compromiso ganglionar

demostró ser superior a la hormonoterapia sola en cuanto a supervivencia libre de enfermedad invasiva (SLEI) y supervivencia libre de recurrencia a distancia (SLRD), con tasas estimadas de SLEl a 2 años de $92.2 \%$ y $88.7 \%(H R=0.74 ; p=0.0096)$, y tasas estimadas de SLRD de $93.6 \%$ y $90.3 \%$ (HR 0.71; $\mathrm{p}=0.0085$ ), para los grupos con y sin amebaciclib, respectivamente $(p<0.01)$. El $16.6 \%$ de los participantes suspendieron el amebaciclib debido a eventos adversos (32).

Los autores de este estudio ponen énfasis en la importancia de sus resultados, considerando que en los últimos 20 años hay pocos avances en cuanto a adyuvancia hormonal en tumores RH+, HER2-. Los resultados son aun más alentadores si se considera el subgrupo de pacientes premenopáusicas, en donde la disminución del riesgo de recaer en los 2 primeros años fue 37\% (HR 0.63; IC 95\% 0.44-0.92). Dado que las recaídas tempranas se correlacionan con resistencia endocrina primaria, el abemaciclib se convierte en una excelente estrategia para superarla (32).

Finalmente, el estudio PALLAS (PALbociclib CoLlaborative Adjuvant Study) (33) aleatorizó 5.760 pacientes con cáncer de mama RH+, HER2-, estadios
II y III a recibir terapia hormonal más palbociclib vs. terapia hormonal sola. Con un seguimiento a casi 24 meses, la tasa de SLEl fue $88.2 \%$ en el grupo de la combinación vs. $88.5 \%$ en el grupo control $(H R=0.93 ; p=0.51)$. La SLRD fue $89.3 \%$ y $90.7 \%$ (HR $1.00 ; p=0.997)$, respectivamente. No se observó ninguna diferencia en el subgrupo de pacientes de alto riesgo. Para resaltar, se observó que el $42 \%$ de los participantes suspendieron el palbociclib debido a eventos adversos, el $64 \%$ de ellos por toxicidad inaceptable (33). Existen otros estudios en curso de manejo adyuvante con iCDK4/6, pero sus resultados oficiales aún no han sido publicados $(34,35)$.

Las Tablas 1 y 2 resumen los resultados de los estudios clínicos más relevantes del manejo hormonal adyuvante en pacientes premenopáusicas y posmenopáusicas con cáncer de mama $\mathrm{RH}+$.

Abordaje terapéutico adoptado por las Unidades Funcionales de Oncología Clínica y Mama y Tejidos Blandos del INC

Teniendo en cuenta que la mayoría de las pacientes que ingresan a la Unidad Funcional de Mama y Tejidos 
Blandos se presentan con cáncer de mama en estados clínicos avanzados (50\%), y que el porcentaje de recidiva tumoral es mayor en aquellas con tumores luminal B-HER2 negativo, así como en quienes presentan enfermedad residual pos quimioterapia neoadyuvante
(36), se tomó la decisión de estandarizar el manejo hormonal adyuvante de las pacientes de acuerdo al riesgo de recidiva tumoral y a su estado menopáusico (premenopáusicas y posmenopáusicas). Figuras 1 y 2.

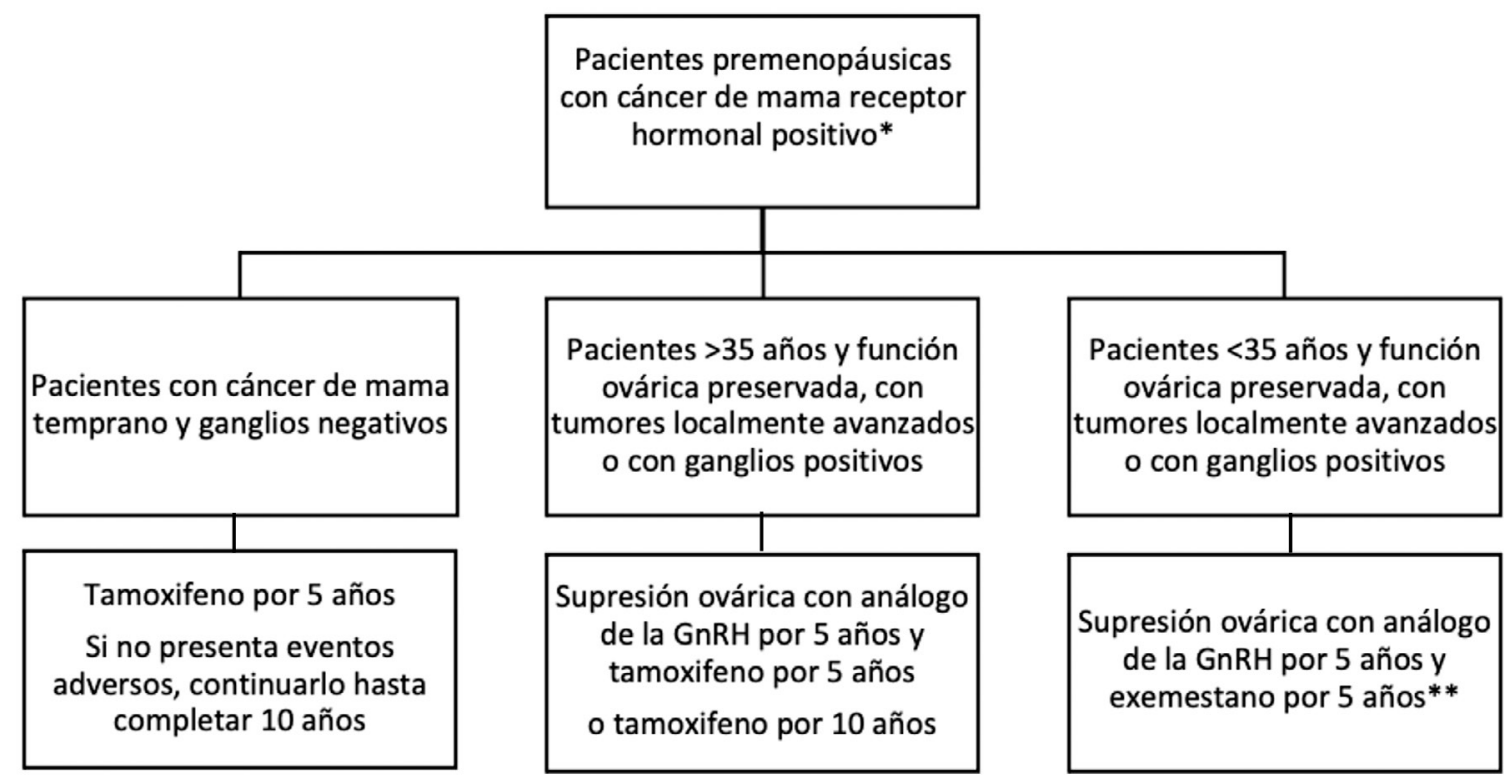

Figura 1. Flujograma de tratamiento adyuvante en pacientes premenopáusicas.

* Si la paciente se torna menopáusica durante la adyuvancia hormonal, continuar el manejo según figura No. 2

** Realizar pruebas de función ovárica cada 3 meses para verificar el bloqueo de la función ovárica. De no poder garantizar el bloqueo hormonal, tamoxifeno por 10 años

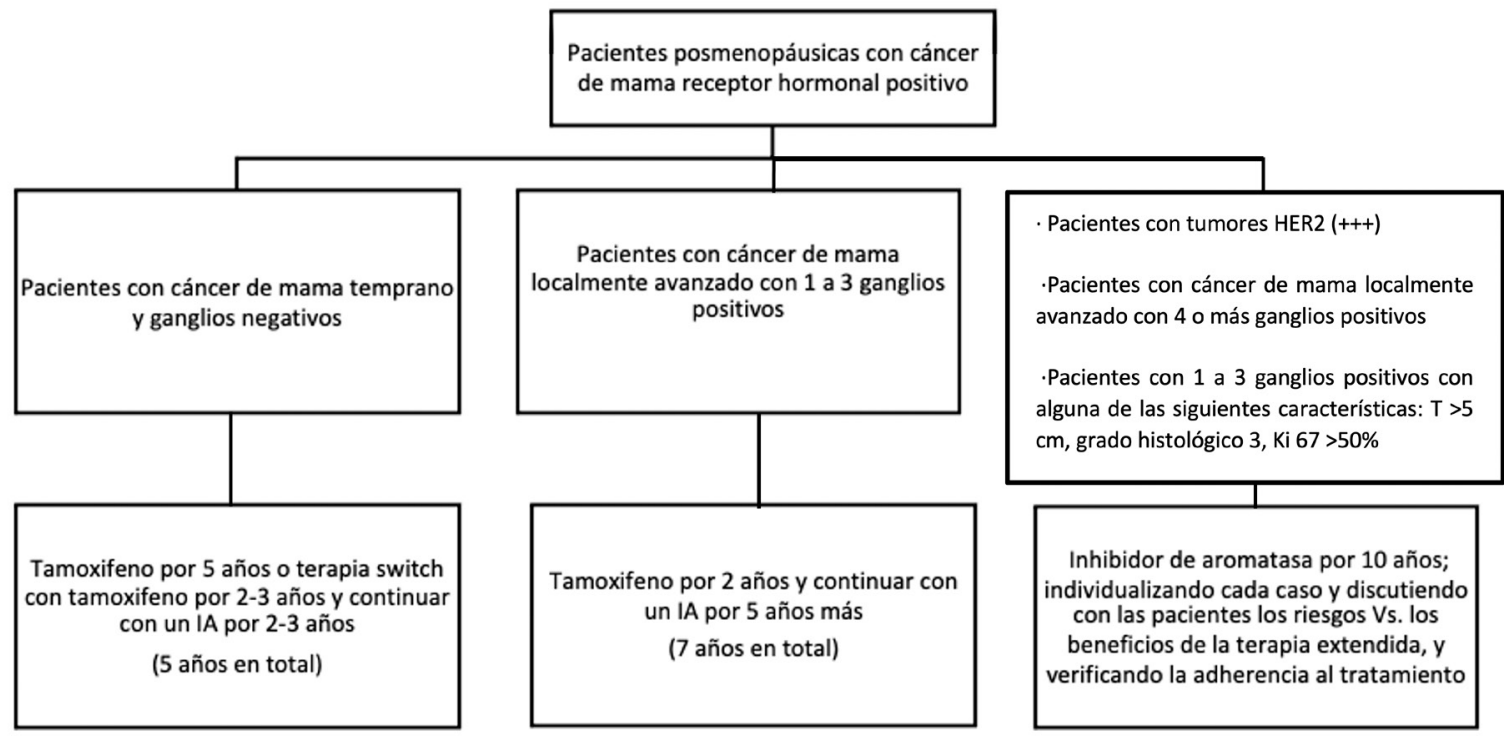

Figura 2. Flujograma de tratamiento adyuvante en pacientes posmenopáusicas. 


\section{Responsabilidades éticas}

\section{Protección de personas y animales.}

Los autores declaran que, por ser este un artículo de revisión de evidencia científica, no hay pacientes involucrados.

\section{Derecho a la privacidad y consentimiento informado.}

No ha sido necesario obtener consentimientos informados.

\section{Fuente de financiación}

\section{Ninguna.}

\section{Conflicto de intereses}

Ninguno de los autores tiene conflictos de interés.

\section{Referencias}

1. Waks AG, Winer EP. Breast Cancer Treatment: A Review. JAMA. 2019 Jan 22;321(3):288-300. doi: 10.1001/jama.2018.19323. PMID: 30667505. https://doi.org/10.1001/jama.2018.19323

2. Guía de Práctica Clínica (GPC) para la detección temprana, tratamiento integral, seguimiento y rehabilitación de pacientes con cáncer de mama. Sistema de Seguridad Social, Colombia, 2013. Guía No. 19.

3. Pan H, Gray R, Braybrooke J, et al. 20-Year Risks of BreastCancer Recurrence after Stopping Endocrine Therapy at 5 Years. N Engl J Med 2017; 377:1836-1846. https://doi.org/10.1056/ NEJMoa1701830

4. Goldvaser H, Barnes TA, Šeruga B, et al. Toxicity of Extended Adjuvant Therapy With Aromatase Inhibitors in Early Breast Cancer: A Systematic Review and Meta-analysis. JNCI: Journal of the National Cancer Institute, Volume 110, Issue 1, January 2018, Pages 31-39. https://doi.org/10.1093/jnci/djx141

5. Early Breast Cancer Trialists' Collaborative Group (EBCTCG). Tamoxifen for early breast cancer: an overview of the randomised trials. Lancet 1998; 351: 1451-67.

6. Early Breast Cancer Trialists' Collaborative Group (EBCTCG). Relevance of breast cancer hormone receptors and other factors to the efficacy of adjuvant tamoxifen: patient-level meta-analysis of randomised trials. Lancet 2011; 378: 771-84. https://doi.org/10.1016/S0140-6736(11)60993-8

7. Davies C, Pan H, Godwin J, et al. Long-term effects of continuing adjuvant tamoxifen to 10 years versus stopping at 5 years after diagnosis of oestrogen receptor-positive breast cancer: ATLAS, a randomised trial. Lancet 2013; 381: 805-16. https://doi. org/10.1016/S0140-6736(12)61963-1
8. Gray R, Rea D, Handley K, et al. aTTom: long-term effects of continuing adjuvant tamoxifen to 10 years versus stopping at 5 years in 6934 women with early breast cancer. J Clin Oncol 2013; 31 (suppl): abstr 5.

9. M P.A. Francis, O. Pagani, G.F. Fleming, B.A, et al. Tailoring Adjuvant Endocrine Therapy for Premenopausal Breast Cancer. N Engl J Med; June 4, 2018, at NEJM.org. https://doi. org/10.1056/NEJMoa1803164

10. Early Breast Cancer Trialists' Collaborative Group (EBCTCG). Aromatase inhibitors versus tamoxifen in early breast cancer: patient-level meta-analysis of the randomised trials. Lancet 2015; 386: 1341-52. https://doi.org/10.1016/S01406736(15)61074-1

11. Benson J, Jatoi I. Extended endocrine therapy in early breast cancer: how long and who for?. Future Oncol. (2019) 16(1), 4327-4336. https://doi.org/10.2217/fon-2019-0254

12. Goss F, Ingle J, Martino S et al. Randomized trial of letrozole in post-menopausal women after 5 years of tamoxifen therapy for early stage breast cancer. N. Eng. J. Med. 349, 1793-1802 (2003). https://doi.org/10.1056/NEJMoa032312

13. Goss $\mathrm{P}$, Mamounas $\mathrm{E}$, Jakesz $\mathrm{R}$ et al. Aromatase inhibitors versus not (placebo/observation) as late extended adjuvant therapy for postmenopausal women with early stage breast cancer: overviews of randomized trials of aromatase inhibitors after approximately 5 years of tamoxifen. Cancer Res. 69, 4081 (2009).

14. Goss $\mathrm{PE}$, Ingle JN, Pritchard $\mathrm{KI}$ et al. Extending aromatase inhibitor adjuvant therapy to 10 years. N Engl J Med 375, 209219 (2016). https: / /doi.org/ 10.1056/NEJMoa1604700

15. Jakesz R, Greil R, Gnant M, et al. Extended Adjuvant Therapy With Anastrozole Among Postmenopausal Breast Cancer Patients: Results From the Randomized Austrian Breast and Colorectal Cancer Study Group Trial 6a. J Natl Cancer Inst 2007;99:1845-53. https://doi.org/10.1093/jnci/djm246.

16. Mamounas E, Jeong JH, Wickerham DL. Benefit From Exemestane As Extended Adjuvant Therapy After 5 Years of Adjuvant Tamoxifen: Intention-to-Treat Analysis of the National Surgical Adjuvant Breast and Bowel Project B-33 Trial. J Clin Oncol 2008, 26:1965-1971. https://doi.org/10.1200/JCO.2007.14.0228.

17. Blok E, Kroep JR, Meershoek-Klein Kranenbarg E et al. Optimal duration of extended adjuvant endocrine therapy for early breast cancer: results of the IDEAL trial (BOOG 2006-05). J. Natl Cancer Inst. 110 (1) https://doi.org/10.1093/jnci/djx134

18. Tjan-Heijnen VCG, van Hellemond IEG, Peer PGM et al. Extended adjuvant aromatase inhibition after sequential endocrine therapy (DATA): a randomized Phase III trial. Lancet Oncol. 18(11), 1502-1511 (2017). https://doi.org/ 10.1016/ $\underline{\mathrm{S} 1470-2045(17) 30600-9}$

19. Mamounas E, Bandos H, Lembersky B et al. Use of letrozole after aromatase inhibitor-based therapy in post-menopausal breast cancer (NRG Oncology/NSABP B-42): a randomized, double blind placebo-controlled, Phase III trial. Lancet 20(1), 88-99 (2019). https://doi.org/10.1016/S1470-2045(18)30621-1

20. Gnant $M$, Steger $G$, Greil $R$ et al. A prospective randomized multicentre Phase-III trial of additional 2 versus additional 5 
years of anastrozole after initial 5 years of adjuvant endocrine therapy - results from 3,484 post-menopausal women in the ABSCSG-16 trial. San Antonio Breast Cancer Symposium, San Antonio, TX, USA, 7 December 2017.

21. Ohtani $\mathrm{S}$, lijima $\mathrm{K}$, Higaki $\mathrm{K}$ et al. A prospective randomized multicenter open-label Phase III trial of extending aromatase inhibitor therapy to 10 years: results from 1,697 postmenopausal women in the N-SA BC05 trial: arimidex extended adjuvant randomized study (AERAS). San Antonio Breast Cancer Symposium San Antonio, TX, USA, 4-8 December 2018. https:// doi.org/10.1158/1538-7445.SABCS18-GS3-04

22. Del Mastro L, Mansutti M, Bisagni G, Ponzone R, Durando A, Amadducci L. Benefit from letrozole as extended adjuvant therapy after sequential endocrine therapy: a randomized Phase III study of Gruppo Italiano Mammella (GIM). J. Clin. Oncol. 37(Suppl. 1), Abstr 504 (2019).

23. Duffy SW, Vulkan D, Cuckle $\mathrm{H}$, et al. Extended adjuvant intermittent letrozole versus continuous letrozole in postmenopausal women with breast cancer (SOLE): a multicentre, open-label, randomised, phase 3 trial. www. thelancet.com/oncology Published on line, November 17, 2017. https://doi.org/10.1016/S1470-2045(17)30715-5

24. Finn RS, Martin M, Rugo HS, Jones S, Im SA, Gelmon K, et al. Palbociclib and letrozole in advanced breast cancer. $\mathrm{N}$ Engl J Med. 2016;375(20):1925-36. https://doi.org/10.1056/ NEJMoa1607303

25. Hortobagyi GN, Stemmer SM, Burris HA, Yap YS, Sonke GS, PaluchShimon S, et al. Ribociclib as first-line therapy for HR-positive, advanced breast cancer. N Engl J Med. 2016;375(18):1738-48. https://doi.org/10.1056/NEJMoa1609709

26. Goetz MP, Toi M, Campone M, Trédan O, Bourayou N, Sohn J, et al. MONARCH 3: Abemaciclib as initial therapy for advanced breast cancer. J Clin Oncol. 2017;35(32):3638-46. https://doi. org/10.1200/JCO.2017.75.6155

27. Tripathy D, Im SA, Colleoni M, Franke F, Bardia A, Harbeck N, et al. Ribociclib plus endocrine therapy for premenopausal women with hormone-receptor-positive, advanced breast cancer (MONALEESA-7): a randomised phase 3 trial. Lancet Oncol [Internet]. 2018;19(7):904-15. Available from: http://dx.doi. org/10.1016/S1470-2045(18)30292-4.

28. Im SA, Lu YS, Bardia A, Harbeck N, Colleoni M, Franke F, et al. Overall survival with ribociclib plus endocrine therapy in breast cancer. N Engl J Med. 2019;381(4):307-16. https://doi. org/10.1056/NEJMoa1903765
29. Turner NC, Slamon DJ, Ro J, Bondarenko I, Im SA, Masuda N, et al. Overall survival with palbociclib and fulvestrant in advanced breast cancer. N Engl J Med. 2018;379(20):1926-36. https:// doi.org/10.1056/NEJMoa1810527

30. Slamon DJ, Neven P, Chia S, Fasching PA, De Laurentiis M, Im $\mathrm{SA}$, et al. Overall survival with ribociclib plus fulvestrant in advanced breast cancer. N Engl J Med. 2020;382(6):514-24. https://doi.org/10.1056/NEJMoa1911149

31. Sledge GW, Toi M, Neven P, Sohn J, Inoue K, Pivot X, et al. The Effect of Abemaciclib Plus Fulvestrant on Overall Survival in Hormone Receptor-Positive, ERBB2-Negative Breast Cancer That Progressed on Endocrine Therapy - MONARCH 2: A Randomized Clinical Trial. JAMA Oncol. 2020;6(1):116-24. https://doi. org/10.1001/jamaoncol.2019.4782

32. Johnston Stephen R. D, Harbeck Nadia, Hegg T Roberto, et al. Abemaciclib Combined With Endocrine Therapy for the Adjuvant Treatment of HR+, HER2-, Node-Positive, High-Risk, Early Breast Cancer (monarchE). ascopubs.org/journal/ jco on September 20, 2020: DOI https://doi. org/10.1200/JCO.20. 02514. https://doi.org/10.1200/JCO.20.02514

33. Mayer EL, Gnant MI, DeMichele A, et al. PALLAS: A randomized phase trial of adjuvant palbociclb with endocrine therapy vs andocrine therapy alone for HR+/HER2 early breast cancer. ESMO Virtual Congress 2020. Abstract LBA 12. Presented September 20, 2020.

34. Slamon DJ, et al. NATALEE: Phase III study of ribociclib (RIBO) + endocrine therapy (ET) as adjuvant treatment in hormone receptor-positive $(\mathrm{HR}+)$, human epidermal growth factor receptor 2-negative (HER2-) early breast cancer (EBC). May 2019. Journal of Clinical Oncology 37(15_suppl):TPS597TPS597. DOI: 10.1200/JCO.2019.37.15_suppl.TPS597.

35. Von Minckwitz G, et at. Abstract OT2-6-11: PENELOPE: Phase III study evaluating palbociclib (PD-0332991), a cyclin-dependent kinase (CDK) 4/6 inhibitor in patients with hormone-receptorpositive, HER2-normal primary breast cancer with high relapse risk after neoadjuvant chemotherapy (GBG-78/BIG1-13). Cancer Res 2013;73(24 Suppl): Abstract nr OT2-6-11.

36. Díaz-Casas SE, Castilla-Tarra JA, Pena-Torres E, et al. Pathological Response to Neoadjuvant Chemotherapy and the Molecular Classification of Locally Advanced Breast Cancer in a Latin American Cohort. The Oncologist 2019;24:1-11. https:// doi.org/10.1634/theoncologist.2019-0300 